\title{
Numerical investigation of some stationary solutions of the Carleman system
}

\author{
Olga Vasil'eva ${ }^{1, *}$ \\ ${ }^{1}$ Moscow State University of Civil Engineering, 26 Yaroslavskoe shosse, Moscow, 129337, Russia
}

\begin{abstract}
The negative stationary solutions of the boundary value problem for the Carleman system of equations are investigated. The kinetic Carleman system is a system of two nonlinear partial differential equations. The system describes the interaction of transportation and nonlinear processes. So, it is used for mathematical modelling of problems in various fields: the kinetic theory of gasses, the gas dynamics, the chemistry, ecology, acoustics etc. In particular, the system can be used to describe autokatalys problems for research of building materials. We present and discuss results of numerical investigation of negative problem solution for different values of parameters. There are three problem parameters domains. For the first parameters domain the stationary solution has stable character, for the second parameters domain the stationary solution has stochastic character and for third domain the stationary solution has unstable character.
\end{abstract}

\section{Introduction}

We consider the initial boundary problem for the kinetic Carleman system of equations. This system can be used for mathematical modelling problems of the kinetic theory of gasses, chemistry and in particular, for research of building materials. So, the investigation of the Carleman system is very interested now [1-5]. One of the important properties of the initial boundary problem for Carleman system is the stability of the stationary solutions. We consider the negative stationary solutions of the initial boundary problem for the Carleman system equation. The finite-difference methods are used for the investigation of the stationary solution properties.

\section{Formulation of the problem}

We consider the initial boundary problem for the Carleman system

$$
\begin{aligned}
& u_{t}+u_{x}=\varepsilon^{-1}\left(w^{2}-u^{2}\right), \quad t>0,0<x<1, \\
& w_{t}-w_{x}=\varepsilon^{-1}\left(u^{2}-w^{2}\right),
\end{aligned}
$$

with initial and boundary conditions

\footnotetext{
* Corresponding author: vasilijeva.ovas@yandex.ru
} 


$$
\begin{gathered}
\left.u\right|_{t=0}=u^{0}(x),\left.w\right|_{t=0}=w^{0}(x) \\
u(t, 0)=u_{0}(t), w(t, 1)=w_{1}(t), u_{0}(0)=u^{0}(0), w_{1}(0)=w^{0}
\end{gathered}
$$

Here $\varepsilon>0$ is the first constant parameter problem. The second parameter of problem we denote by $c<0$. The function $u^{\mathrm{s}}(t, x)=u^{\mathrm{s}}(x)=c$ and $w^{\mathrm{s}}(t, x)=w^{\mathrm{s}}(x)=c$ is the stationary solution (1) with following initial and boundary conditions $u^{0}(x)=c, w^{0}(x)=c, u_{0}(t)=c$, $w_{1}(t)=c$ for any $\varepsilon>0$ and $c<0$.

For the investigation properties of the stationary solutions we consider problem (1) with pertrubated initial condition

and boundary conditions

$$
u^{0}(x)=c, w^{0}(x)=c+\alpha \sin (2 \pi x)
$$

$$
u_{0}(t)=c, w_{0}(t)=c .
$$

Denote the energy of pertrubation of stationary solution $u^{\mathrm{s}}(x), w^{\mathrm{s}}(x)$ by

$$
\begin{aligned}
& e_{1}(t)=\left(\int_{0}^{1}\left(u(t, x)-u^{s}(x)\right)^{2} d x\right)^{\frac{1}{2}} \\
& e_{2}(t)=\left(\int_{0}^{1}\left(w(t, x)-w^{s}(x)\right)^{2} d x\right)^{\frac{1}{2}}
\end{aligned}
$$

and total enargy by

$$
E(t)=\left(e_{1}^{2}(t)+e_{2}^{2}(t)\right)^{0.5}
$$

\section{Results of numerical investigation}

At first we consider the problem (1) - (3) for the $c=-0.1, \varepsilon=0.6$ and $\alpha=0.1$. Figure 1 shows the dependences of the energy of pertrubation of stationary solution $e_{1}(t), e_{2}(t)$. Figure 2 presents the total perturbation energy, which tends to zero. Figure 3 presents phase plane $\left(e_{1}, e_{2}\right)$. So we can see the stabilization the perturbation solution to stationary solution.

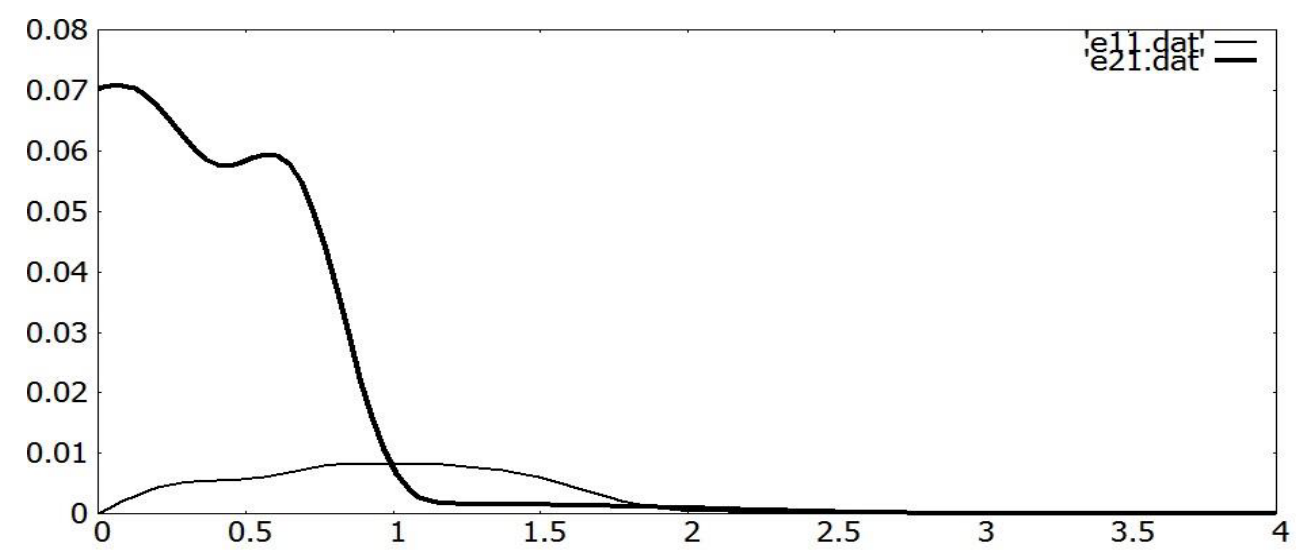

Fig. 1. Energy of perturbations. 


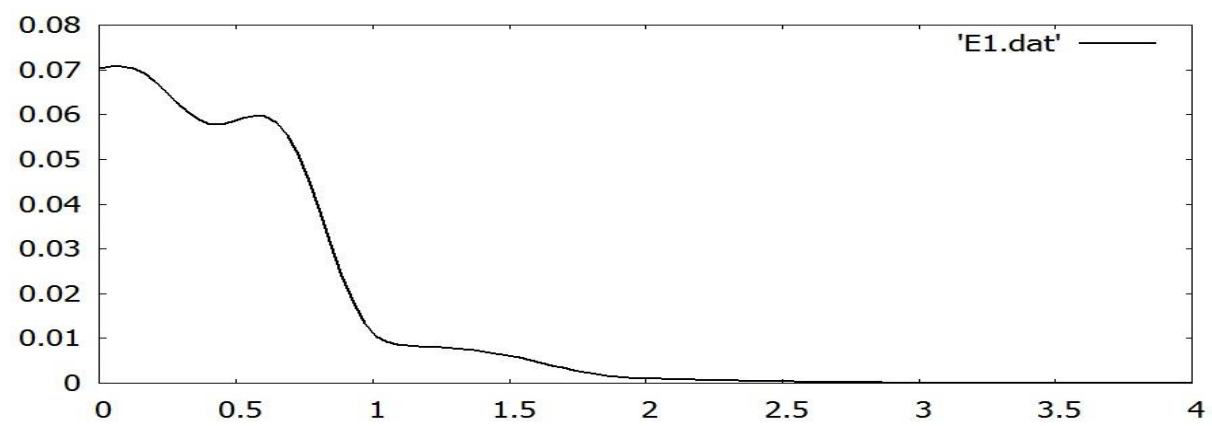

Fig.2. Total energy of perturbation.

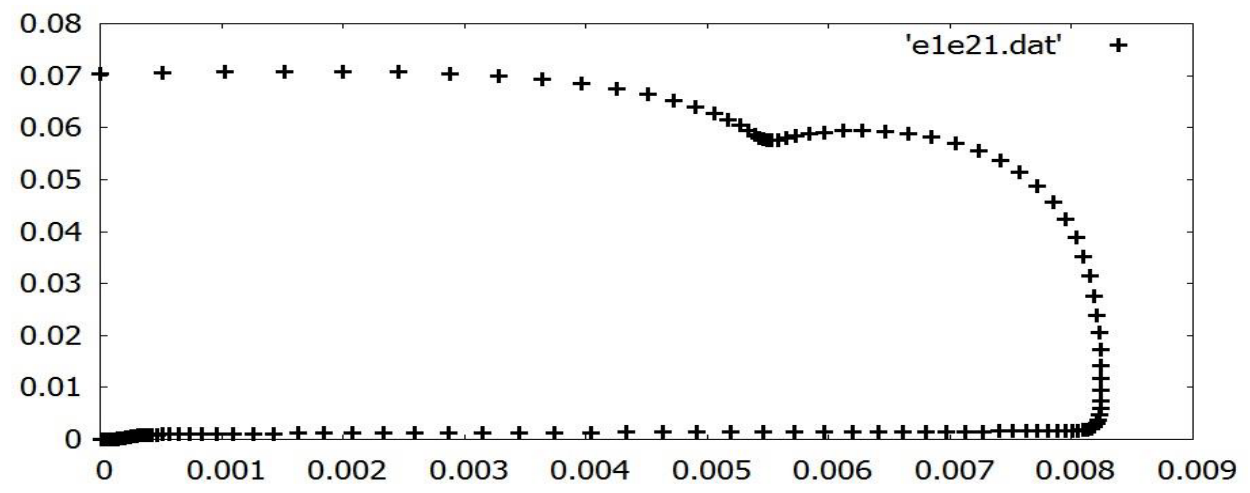

Fig. 3. Phase plane.

Figures 4 - 8 present results of numerical investigation of problem (1) - (3) for the $c=-$ $0.5, \varepsilon=0.6$ and $\alpha=0.1$. Figure 4,5 show numerical solution of problem $u(t, x)$ and $w(t, x)$ for the values of the time variable $t_{0}=0, t_{1}=0.8, t_{2}=1.6, t_{3}=2.4, t_{4}=3.2, t_{5}=4$. Figures 6,7 present the dependences of the energy of pertrubation of stationary solution $e_{1}(t), e_{2}(t)$ and total energy $E(t)$. Dependences have oscillatory nature. Figure 8 presents phase plane $\left(e_{1}, e_{2}\right)$.

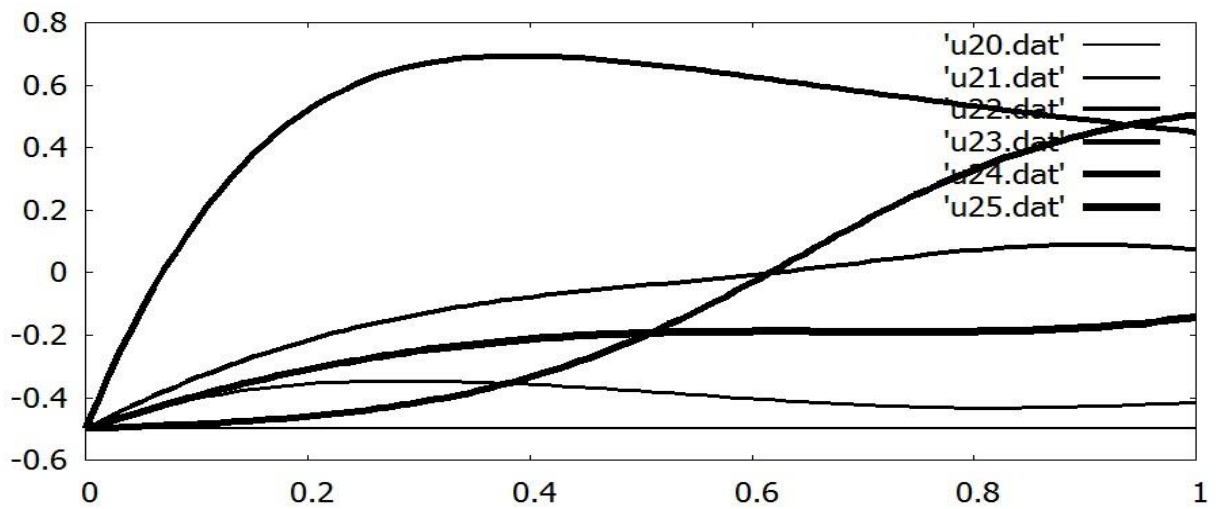

Fig. 4. Numerical solution of problem $u(t, x)$. 


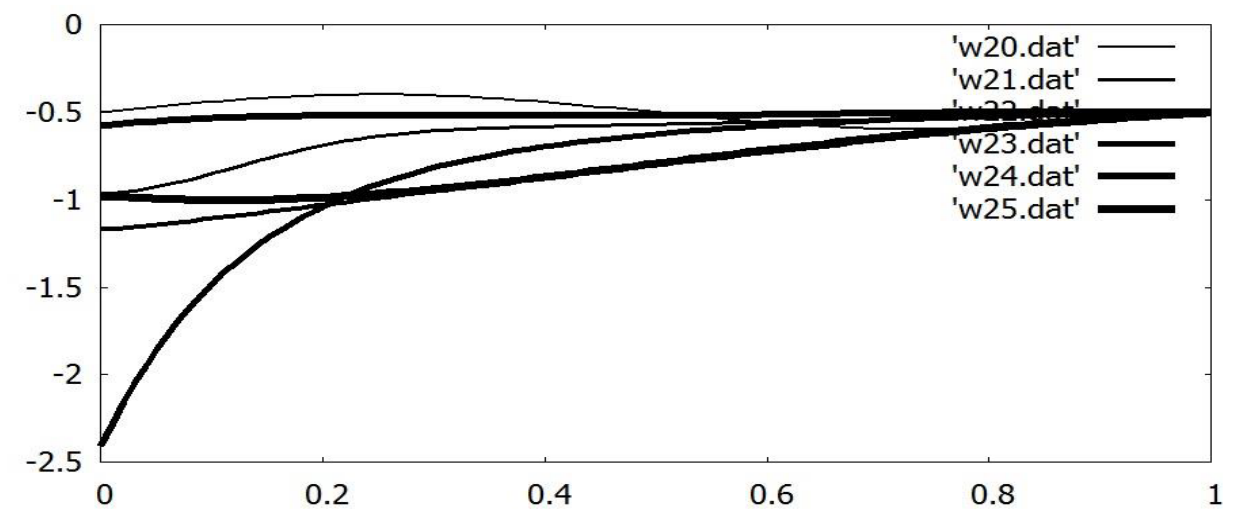

Fig. 5. Numerical solution of problem $w(t, x)$.

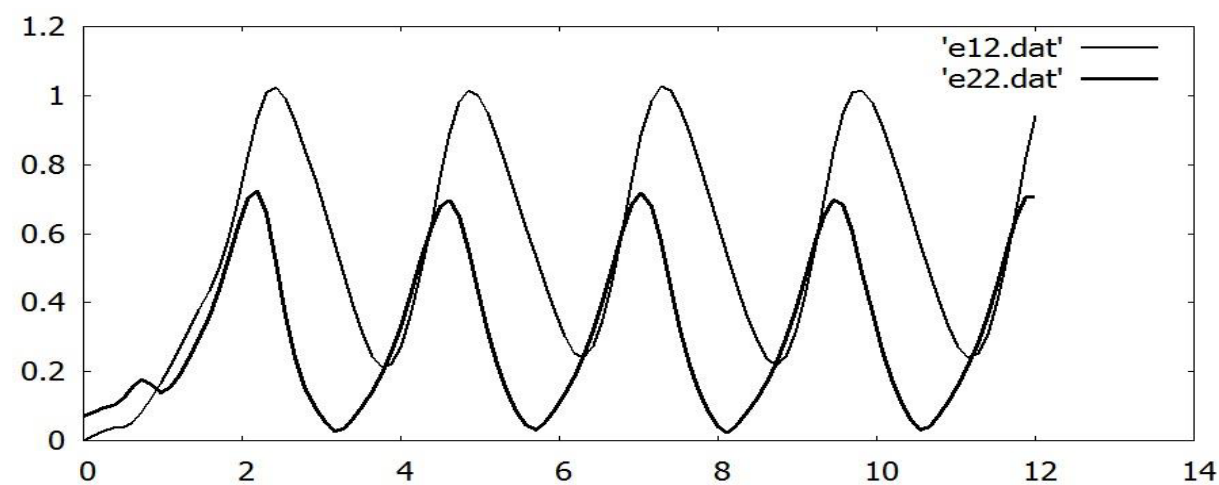

Fig. 6. Energies of perturbations.

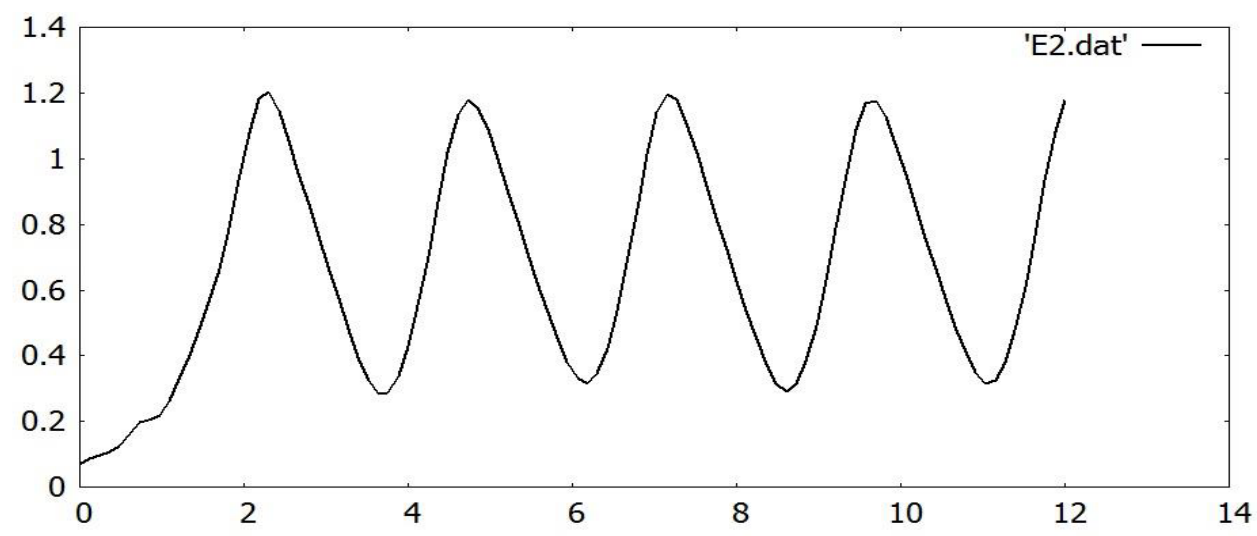

Fig. 7. Total energy 


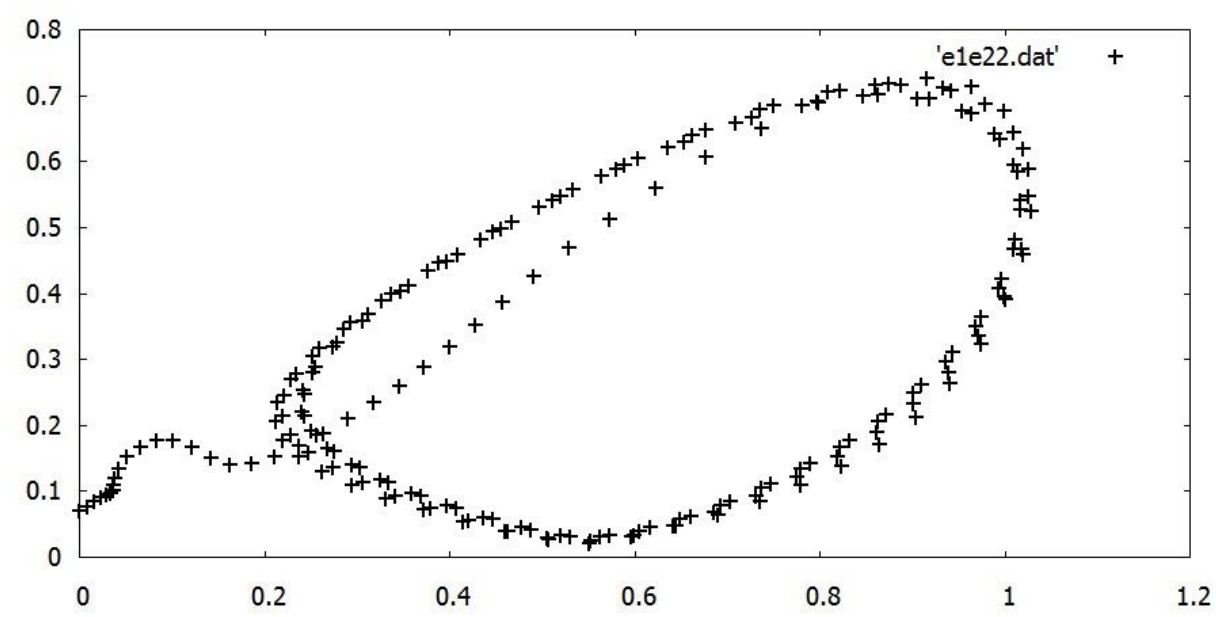

Fig. 8. Phase plane

Figures 9-11 present results of numerical investigation of the problem (1) - (3) for $c=-1$, $\varepsilon=0.6$ and $\alpha=0.00001$. Here we can see energy increasing for small perturbation of the stationary solution $(\alpha=0.00001)$.

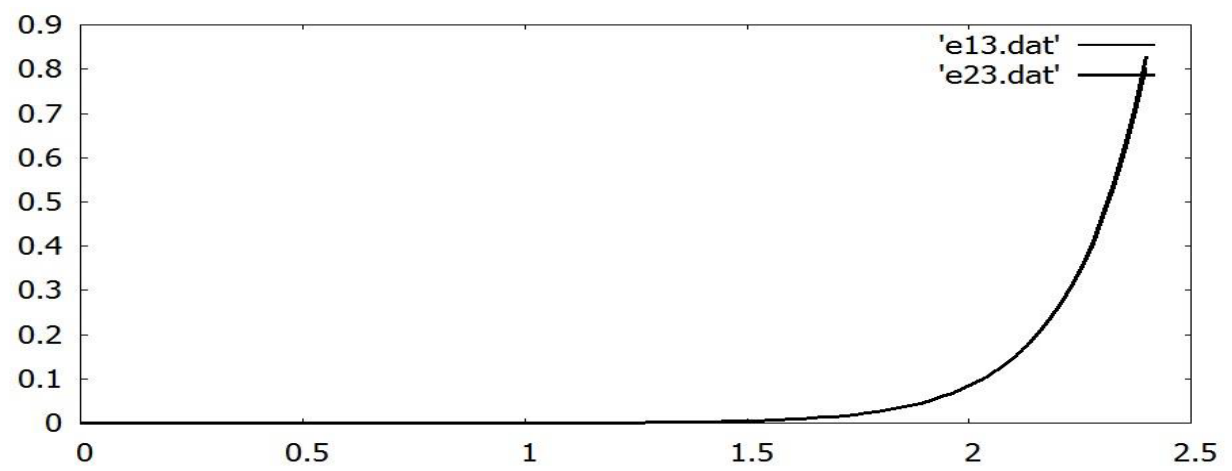

Fig. 9. Energies of perturbations.

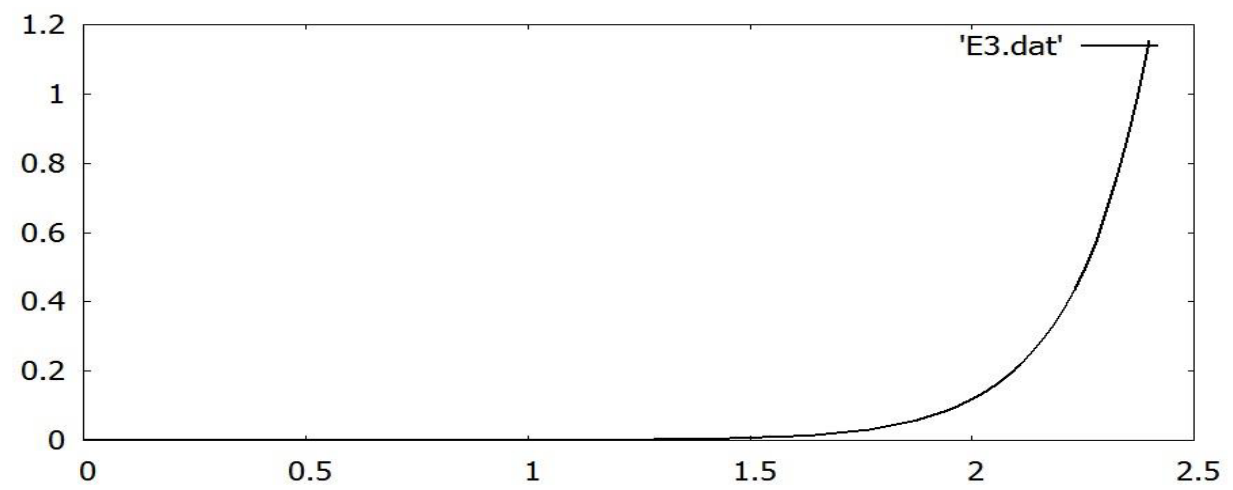

Fig. 10. Total energy. 


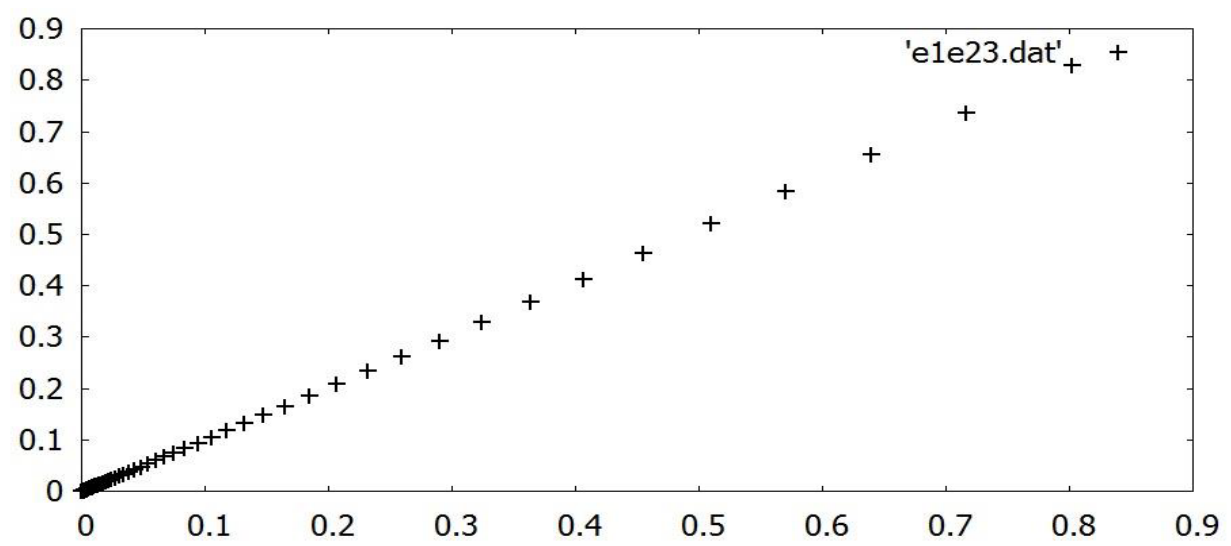

Fig. 11. Phase plane.

\section{Conclusion}

We present the results of numerical investigation of the perturbed stationary solution of the initial boundary problem for the Carleman system. Based on numerical investigation we can conclude that for some fixed $\varepsilon$ there are three different kind of solution behavior. A perturbation of negative stationary solution close to zero leads to stabilization of the perturbed solution to the equilibrium state. With decreasing values of the parameter we have a chaotic nature of perturbed solution. With a further decrease of the parameter $c$, we can see the instability of the perturbed solution. The author would like to express gratitude to Professor E.V.Radkevich for formulation of the problem and useful discussions and comments.

\section{References}

1. S. K. Godunov, U. M. Sultangazin, Math. Surv. 26, 3 (1971)

2. E. V. Radkevich, J. Math. Sci. 181, 5 (2012)

3. E. V. Radkevich, J. Math. Sci. 184, 4 (2012)

4. V. V. Aristov, O.V. Ilyin, Phys. Let. A. 374, 4381-4384 (2010)

5. E. V. Radkevich, O. A.Vasil'eva, S. A.Duhnovskij, J. Math. Sci. 201, 32 (2015) 